\title{
Hypertricosis overlying plexiform neurofibroma in a female patient: a rare presentation
}

\author{
Faizan Younus Shah", Mohammad Abid Keen², Ifrah Shafat Kitab³, Aaqib Aslam Shah¹ \\ ${ }^{1}$ Department of Dermatology, STDs and Leprosy, Government Medical College, Srinagar, Jammu and Kashmir, India, \\ ${ }^{2}$ Directorate of Health Services, Srinagar, Jammu and Kashmir, India, ${ }^{3}$ Department of Ophthalmology, Government Medical \\ College, Srinagar, Jammu and Kashmir, India
}

Corresponding author: Faizan Younus Shah, MD, E-mail: faizanyounis@gmail.com

\begin{abstract}
Periorbital neurofibromas can occur either in isolation or in association with neurofibromatosis (NF). Neurofibromas in the periorbital region account for $0.5-2.4 \%$ of orbital tumours. Hypertrichosis overlying neurofibromas are rarely reported cases in literature. We report a case of localized hypertrichosis overlying neurofibroma in a 27 year old female with no other features of neurofibromatosis. Neurofibromas can occur as solitary lesions or in association with neurofibromatosis. Our patient had diffuse hypertrichosis on a hyperpigmented macule overlying and around the periorbital neurofibroma.
\end{abstract}

Key words: Plexiform; Neurofibroma; Hpertrichosis

\section{INTRODUCTION}

Periorbital neurofibromas can occur either in isolation or in association with neurofibromatosis (NF). Neurofibromas in the periorbital region account for 0.5 $2.4 \%$ of orbital tumours [1]. Pigmented neurofibromas $(\mathrm{PNF})$ are rare tumours laden with melanin containing pigmented cells, and account for less than $1 \%$ of all neurofibromas [2]. PNF's can occur in patients with or without neurofibromatosis. Hypertrichosis is defined as non-androgen dependent excessive growth of hair that is abnormal for the age, gender or race of an individual or for that particular area of the body. Hypertrichosis overlying neurofibromas are rarely reported cases in literature [3]. We report a case of localized hypertrichosis overlying neurofibroma in a 27 year old female with no other features of neurofibromatosis.

\section{CASE REPORT}

A 27 year old woman presented with a swelling involving the right upper side of the face. The swelling had been present since childhood as far as the patient could remember. However, it could not be ascertained with surety whether the swelling had been present from birth or not despite repeated questioning from various family members. The medical history of the patient was rather uneventful. Her growth and development had been normal and comparable with peer groups. The swelling was asymptomatic upto a year back, when the patient started to notice a gradual increase in the size of the swelling. There was no history of neurofibromatosis (NF) in the family. On examination, a light brown to brownish black hyperpigmented macule with hypertrichosis overlying a swelling was noted in the right periorbital region (Fig. 1). The horizontal extension of the swelling was from the nose in the midline upto the right ear. A rough guide to the vertical extension of the swelling would be from the parietotemporal line and mid forehead above upto the Frankfurt plane below. The hyperpigmentation and hypertrichosis extended beyond the margins of the swelling. The hypertrichosis was most prominent in the mid facial area while the pigmentation extended upto the mandible. The swelling, at the time of examination, resulted in partial closure of the right eye, obstructed

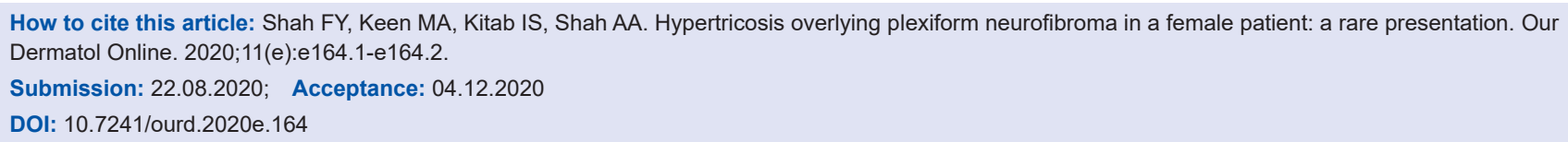




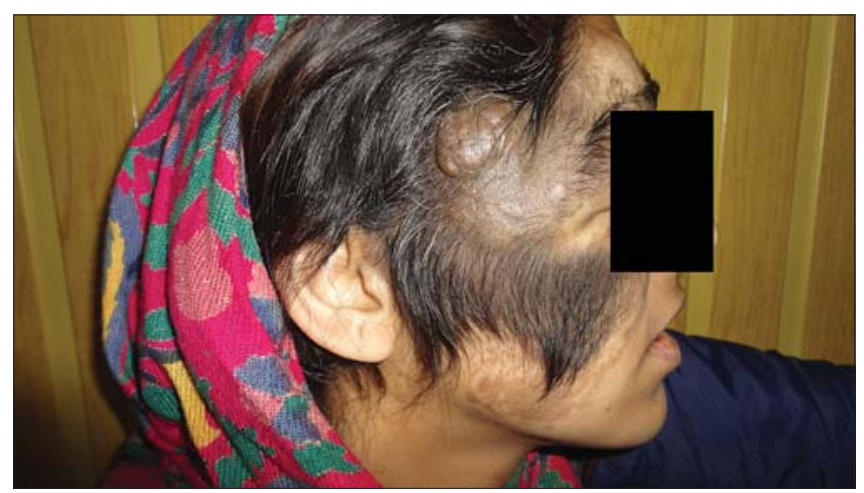

Figure 1: Hyperpigmented macule with hypertrichosis overlying a swelling in the right periorbital region.

the vision of the patient and resulted in complaints of diplopia. However, the visual acuity after manual elevation of the swelling was 6/6 in both eyes and the diplopia was also alleviated. On palpation, two distinct subcutaneous, soft, non-tender swellings, with a 'bag of worms' like consistency, could be palpated. Pseudo-darier's sign (transient piloerection and elevation or increased induration of a lesion induced by rubbing) [4], used as a clinical diagnostic measure for smooth muscle hamartoma was negative in the patient. No other findings in favour of type 1 or type 2 neurofibromatosis could be documented. There were no café au lait macules, axillary or inguinal freckling, or any other neurofibromas. The ophthalmologist could not detect any Lisch nodules in the iris of the patient. Histopathological examination revealed abundant hair follicles in the dermis and encapsulated spindle cells with S-shaped nuclei within deep reticular dermis and subcutis, consistent with neurofibroma. The patient underwent partial excision of the tumour in the department of plastic surgery for cosmetic reasons.

\section{DISCUSSION}

Neurofibromas can occur as solitary lesions or in association with neurofibromatosis. Our patient had diffuse hypertrichosis on a hyperpigmented macule overlying and around the periorbital neurofibroma. The hyperpigmentation resembled a café-au-lait macule though it is not generally associated with hypertrichosis. Though the patient in our case didn't show other features of neurofibromatosis, the possibility of type 5 neurofibromatosis or segmental neurofibromatosis could not be ruled out. Segmental neurofibromatosis is a mosaic variant of NF characterized by cutaneous lesions limited to a circumscribed body segment, mostly unilateral and only rarely cross the midline [5].

Hypertrichosis in association with neurofibromas is a rarely reported incident in literature [1-3]. A conversion of vellus to terminal hair or a change in the hair-growth cycle are the 2 major mechanism to which hypertrichosis can be attributed. Out of these, the latter may be contributory to the process of hypertrichosis associated with neurofibromas.

\section{CONCLUSION}

This case report documents that hypertrichosis and hyperpigmentation of the periorbital and facial skin can occur in association with an underlying neurofibroma. This is a rare entity and should be kept in differential diagnosis of cases of localized hypertrichosis.

\section{Consent}

The examination of the patient was conducted according to the principles of the Declaration of Helsinki.

The authors certify that they have obtained all appropriate patient consent forms, in which the patients gave their consent for images and other clinical information to be included in the journal. The patients understand that their names and initials will not be published and due effort will be made to conceal their identity, but that anonymity cannot be guaranteed.

\section{REFERENCES}

1. Hassan I, Keen A, Shah PA. Facial plexiform neurofibromatosis in a patient with neurofibromatosis type 1: a case report. Our Dermatol Online. 2012;5:1-4.

2. Inaba M, Yamamoto T, Minami R, Ohbayashi C, Hanioka K. Pigmented neurofibroma: report of two cases and literature review. Pathol Int. 2001;51:565-9.

3. Cho E, Kim H, Lee J, Kim H, Park Y. Localized hypertrichosis overlying neurofibroma. Int J Dermatol. 2012;52:1623-4.

4. Bilgiç Ö, Tunçez akyürek F, Altınyazar HC. Pseudo Darier sign: a distinctive finding for congenital smooth muscle hamartoma. J Pediatr. 2016;169:318.

5. Sobjanek M, Dobosz-kawałko M, Michajłowski I, Pęksa R, Nowicki R. Segmental neurofibromatosis. Postepy Dermatol Alergol. 2014;31:410-2.

Copyright by Faizan Younus Shah, et al. This is an open access article distributed under the terms of the Creative Commons Attribution License, which permits unrestricted use, distribution, and reproduction in any medium, provided the original author and source are credited.

Source of Support: Nil, Conflict of Interest: None declared. 